\title{
Chapter 4 \\ Psychosocial, Physical, and Cognitive Perspectives on the Adolescent Dancer
}

\author{
Siobhan B. Mitchell \\ University of Exeter, $U K$ \\ Lucie Clements \\ The Dance Psychologist, UK
}

\begin{abstract}
Adolescence is a critical period that is heightened for dancers, whether participating recreationally or vocationally. Changes are situated within a highly complex setting and are influenced by many factors, including the dance training environment, personal feelings about changes, and perceptions of changes by significant others. The way in which sub-cultures (such as ballet) construct adolescence is likely to impact upon experiences of, and engagement in, dance, as well as development. The dance context, however, has received little attention in relation to development and maturation from a psychosocial perspective. While the facets of talent that predict engagement or dropout of young dancers have been discussed, little research within dance has viewed the adolescent from a truly developmental perspective. This chapter will explore how contemporary cultural constructions of adolescence apply to the dance context, outline the developmental 'tasks' of adolescence, and discuss how young dancers navigate these tasks, drawing on psychosocial perspectives.
\end{abstract}

\section{INTRODUCTION}

Adolescence is a developmental stage that serves as a transition between childhood and adulthood. Adolescence is both a cultural construction and a dynamic concept which continues to be redefined with growing knowledge. Societal changes over the last 200 years, such as later completion of education and increased full-time employment (Worthman \& Trang, 2018), have altered the conditions for physical development and redefined constructions and experiences of adolescence. Traditionally considered to 
occur between 10 and 18 years of age (e.g. Arnett, 2010), in recognition of the changing demands of adolescence, it was recently defined as the period of age between 10 to 24 years (Worthman \& Trang, 2018). As such, adolescence is a fascinating stage that is the subject of considerable writing by psychologists and sociologists. In dance contexts, it is fundamental that teachers, parents and students alike know the changes and adaptations occurring during this vital phase of a dancer's development.

During adolescence, numerous psychosocial, physical and cognitive changes occur, each posing unique challenges. This uniqueness perhaps explains observed trends of increased mental health concerns (Dahl, 2004) and dropout from schooling and extracurricular activities (Crane \& Temple, 2015), all of which have increased prevalence in females than males (Bor et al., 2014; Dumith et al., 2011). Adding elite training into the developmental cooking pot has implications for the adolescent experience, and the uniqueness of the dance training environment may exacerbate or extend these challenges. Distinct differences in pubertal changes between sexes and subsequent differences in the measurement of pubertal status and timing necessitate consideration of each sex individually. Within the social context of dance, overt pubertal changes carry different social meaning and values for females and male, and thus experiences of puberty are likely to differ between sexes. While recognising the complex challenges for the male adolescent dancer (Clegg et al., 2016), there is a lack of data relating to the growth and maturation of male adolescent dancers, and thus some parts of this chapter will focus on data that relates only to female dancers. Existing research within the social psychology of dance has also tended to recruit entirely female or female dominant samples which do not include sex in the analysis. Thus there is little evidence regarding sex-based differences. In addition, much-existing data relates to ballet dancers specifically, with little data available in other genres. This chapter will review the available literature in this area, noting a bias towards citing samples of female classical ballet dancers.

All in all, various biological, psychological and physical changes occur during adolescence, whereby individual adaptation and environment are significant in determining the direction of outcomes (Dahl, 2004). This chapter explores how contemporary cultural constructions of adolescence apply in dance and discusses how young dancers navigate the psychosocial, cognitive and physical developmental 'tasks' of adolescence. Throughout, the chapter draws on psychosocial perspectives, acknowledging how development may be impacted by or in the dance context. We draw on research regarding sociocultural and maturational constraints on dancer development during adolescence. Finally, we recommend approaches to negotiate the challenges of the adolescent dancer.

\section{BACKGROUND}

Adolescence is a critical period that permeates through all social environments that a young person is involved in and explores; development is relative and reactive to family life, schooling, communities, and increasing relevance, social media, and the internet (Dahl, 2004). The extent to which each environment supports and nurtures the individual impacts on the adolescent experience. Whether participating in dance recreationally or vocationally, changes are situated within a complex, multivariate setting. These changes are influenced by the dance training environment, personal feelings about pubertal changes and perceptions of these changes by significant others, including teachers, parents and peers.

Traditionally, adolescent participation in leisure or extracurricular activity is shown to facilitate positive outcomes, including enhanced academic achievement (Fredricks \& Eccles, 2008). Participation might also develop autonomy, which may, in turn, increase adolescent life satisfaction (Leversen et al., 
2012). Evidence suggests that those from the most autonomy-supportive dance environments are those most likely to display wellbeing, such as the experience of positive affect (e.g. Quested \& Duda, 2009, 2010). Training environments that facilitate the development of individuality, free will, and choice will have preferable emotional and physical consequences, such as greater self-esteem.

Dance, however, often extends beyond being a 'leisure activity'; even when considered 'recreational', adolescent students may spend five or more days a week learning and rehearsing, often resulting in time lost from 'normal' adolescent activities (e.g., Pickard \& Bailey, 2009). For many recreational dancers, weekends are filled with rehearsal and competitions, sometimes even auditions for vocational training. Dance participation may decrease the extent to which an adolescent can make choices because time in dance is at the cost of other activities. Unsurprisingly, this may reduce participation in, or increase dropout from, other extracurricular activities, leading to specialisation in dance. For some, such a demanding schedule results in dropout from dance (Aujla et al., 2015; Vani, Pila \& Sabiston, 2017).

Although the dance environment is comparable to sport and music, it is unique in the interplay of elements of the setting (e.g. mirrors/costuming) and the unique presence of individuals integral to development, such as teachers and peers. Particularly unique to dance training is the setting of learning in a class with peers who are not team-mates (as in some team sport modalities) but are likely to be either implicitly or explicitly perceived as competition for roles or places in a school. Extensive training in these educational or elite training environments can have psychologically negative impacts, encouraging ego orientation (e.g. an extrinsic desire to outperform others), maladaptive perfectionism (Carr \& Wyon, 2003) and anxiety about one's chosen activity (Nordin-Bates et al., 2012). Thus, creating the dance environment is of utmost importance in supporting wellbeing and instilling a healthy mindset. A balanced environment is particularly needed to set an adolescent dancer up for a long career in dance which is likely to prolong exposure to numerous occupational stresses such as career instability, low pay and competition for roles.

In vocational ballet training, the process of puberty (often beginning around age 11 for females and age 12 for males) coincides with increased training intensity and pressure for improvement (Buckroyd, 2000; Pickard, 2015). Young dancers must contend with psychological and social changes (such as renegotiating relationships with parental figures and increasing reliance on peers), and physical changes (such as the growth spurt, breast development and changes in physical proportions and body composition) are particularly likely to have a direct impact on performance and technique (e.g. Beunen et al., 1976; Bowerman et al., 2015). Concurrently, expectations of continued improvement rates remain, and training intensity increases (Buckroyd, 2000). Meanwhile, dancers' personal experiences and feelings about changes are being negotiated, setting the stage for multitudinous challenges when combined with the pressures of dance training environments.

While many recreational dancers drop out during adolescence, the environment might be detrimental to psychological wellbeing for those who enter vocational training (e.g. Anshel, 2004; McEwen \& Young, 2011; Smith, 1998). Ballet training, for example, often appeals to and/or selects those who are delayed in maturation, with psychological consequences for the adolescent. The way dance subcultures construct adolescence is likely to impact upon experiences of, and engagement in, dance and development. Next, these changes from a cultural perspective will be discussed. 


\section{Adolescence as a Cultural Construction}

The period of adolescence encompasses physical, social, emotional and cognitive development, considered to begin aged ten years and span into the early twenties (Worthman \& Trang, 2018). As social and cultural concepts of adult milestones such as finishing education, marriage and becoming a homeowner now occur later, the adolescent period continues to extend (Dahl, 2004; Worthman \& Trang, 2018). Mainly, this is because adolescence is defined by social and cultural notions of what it means to be an 'adult'. While physical development (i.e. puberty) is based within the teenage years, other aspects of development (e.g. cognitive skills) are partially experientially led, and therefore continue developing long after sexual and physical maturation are achieved (Dahl, 2004). In most societies, entry into adolescence coincides with the recognition of puberty, and this transition from childhood to adulthood instigates a social transformation encompassing aspects of social life, including work, greater levels of responsibility and partner choice (Dahl, 2004; Worthman \& Trang, 2018). This concept of social adolescence applies widely and differs by gender and in the level of importance within specific cultural environments (Worthman \& Trang, 2018).

In dance subculture, particularly highly stylised forms such as ballet may hold their notions of social adolescence. For example, in pre-professional ballet training contexts, adolescents are arguably required to display more significant levels of discipline, responsibility and maturity than one might expect from early adolescents outside of this context. In line with broader western culture, expectations for males and females differ distinctly in dance. In ballet, pubertal changes may be welcomed for males, bringing benefits of strength and power, yet seen as detrimental for females, shifting them away from the prescribed ideal of a more prepubescent physique (Buckroyd, 2000; Pickard, 2012).

In the twenty-first century, adolescents' schedules are commonly standardised to progression through schooling and prolonged by expanding prerequisites for adulthood (e.g. school completion, gaining employment/economic foothold, establishing independence), meaning a delay in marriage or starting a family (Worthman \& Trang, 2018). In the dance context, adolescents' schedules are distinct from more standardised schooling routes due to the age of peak performance common to some dance styles. In preprofessional ballet training, for example, pre-pubescence is a developmental stage for the advancement of technical ability and skill and the post-pubertal years are the prescribed stage for a dancer's peak performance (Mitchell et al., in press). Adult roles are assumed both in terms of physical challenge and expected psychosocial and emotional maturity; in other words, adolescence for a pre-professional ballet dancer is cut short. For young dancers delayed in their pubertal development, this poses particular challenges; adjusting to the changes of puberty while auditioning for further training or in the prior years of company life (Mitchell et al., 2017; Mitchell et al., 2020).

In understanding adolescence as a cultural construction, consideration should be given to how adolescence is constructed within the dance subculture. Cultural constructions of adolescence prescribe when adolescence begins and ends, the social milestones that are demonstrative of achieving adult status, and the expectations and norms of events such as puberty (Worthman \& Trang, 2018). Context-sensitive maturational processes at adolescence amplify effects from sociocultural ecology and generate unique opportunities for the biological embedding of culture. Within dance, many adolescent developmental processes may pose challenges to continued, healthy engagement with dance. Physical changes of puberty (e.g. breast development and increased fat mass) may make sport or dance participation less comfortable or appealing to young female participants (Malina, Bouchard \& Bar-Or, 2004). Equally, the cultural feminisation of dance may make participation in dance more challenging for young male participants 
(Clegg et al., 2019). In addition, changes in motivational tendencies as part of psychosocial development can influence a young person's choice to participate (Brown, Patel \& Darmawan, 2017; Dahl, 2004).

The prescribed norms and expectations of the body in ballet are defined by ballet subculture (Pickard, 2015). Various researchers have described this subculture to encompass the ideas, customs, and social behaviour of those involved in the ballet world (e.g. Pickard, 2013, 2015; Benn \& Walters, 2001). Ballet dancers are aware from a young age of the expectations of this subculture and the particular characteristics (physical or otherwise) associated with cultural (physical and/or artistic) 'capital' (Pickard, 2012; Wainwright et al., 2006). The social value of the adolescent body is magnified in its importance as dancers perceive it to have the potential to influence the interaction between dancer and training establishment, as it can ultimately lead to 'rejection' from the system. For example, physical characteristics associated with female maturation (breast development and widening of the hips) appear to hold a lesser social value for significant others within the environment, such as teachers and peers (Mitchell et al., 2016).

The timing of puberty and its associated physical characteristics are essential and, thus far, only studied in females in the context of ballet. Earlier maturation is associated with more significant increases in fat mass and shorter legs relative to a longer torso, and later maturation is associated with smaller gains in fat mass and longer legs relative to a shorter torso (Gay et al., 2014). Within ballet, where it is normative for dancers to mature late, those who mature 'on time' may experience similar psychological disadvantages to early maturing individuals in non-dance populations, such as greater body dissatisfaction and disordered eating behaviours (Brooks-Gunn et al., 1989; Brooks-Gunn \& Warren, 1985; Mendle et al., 2007). The environment created by the dance teacher is influenced by factors such as culturally shared beliefs, attitudes or perceptions concerning puberty and early or late maturation, cultural standards of attractiveness and notions of desirable and undesirable physical development, as shown in Figure 1 (Mitchell et al., 2017, p.296; Mitchell et al., 2020; Petersen \& Taylor, 1980). Attitudes may be favourable towards later maturation due to the likelihood of maintaining a slimmer and more suitable ballet physique (Brooks-Gunn \& Warren, 1985, Mitchell et al., 2016, Mitchell et al., 2020). For females maturing early and in advance of their peers, visible changes associated with puberty may be especially noticeable and conflict with social and cultural norms. While there is no published data on maturation timing in males, likely, maturing early or late would potentially impact physical and/or psychological factors.

Of importance are the reference points and norms available to young dancers. Perceptions of an ideal body are derived from an individual's social context; thus, the formation of ideas of what is healthy and standard will be formulated according to the norms and values of the dance world (Benn \& Walters, 2001). More specifically, while those training in a recreational or non-residential context would inhabit several different 'social worlds' during their adolescence (e.g. school, family home, community context), be exposed to a broader set of norms and ideals. Those training vocationally are likely to be exposed to and subsequently to develop a more narrow set of norms and beliefs about the body and outcomes of puberty. The reference points and norms available to the individual are based on what is expected in ballet rather than the broader social world. The internalisation of these norms and building the self and identity around such specific body expectations may lead to a more negative perception of physical developments (Pickard, 2013).

In sum, dance subcultures construct their notions of cultural adolescence; where norms for the onset of adolescence are prescribed as delayed, social milestones vary significantly from those in everyday western culture, and expectations and norms set for processes such as puberty are distorted with normal processes such as breast development and widening of the hips often stigmatised or seen as abnormal. 
Figure 1. A biocultural model of maturity-associated variation in adolescent dancers. Adapted from dance from Cumming et al. (2012). From "Understanding growth and maturation in the context of ballet: a biocultural approach,” by Mitchell, Haase, Cumming \& Malina, 2017

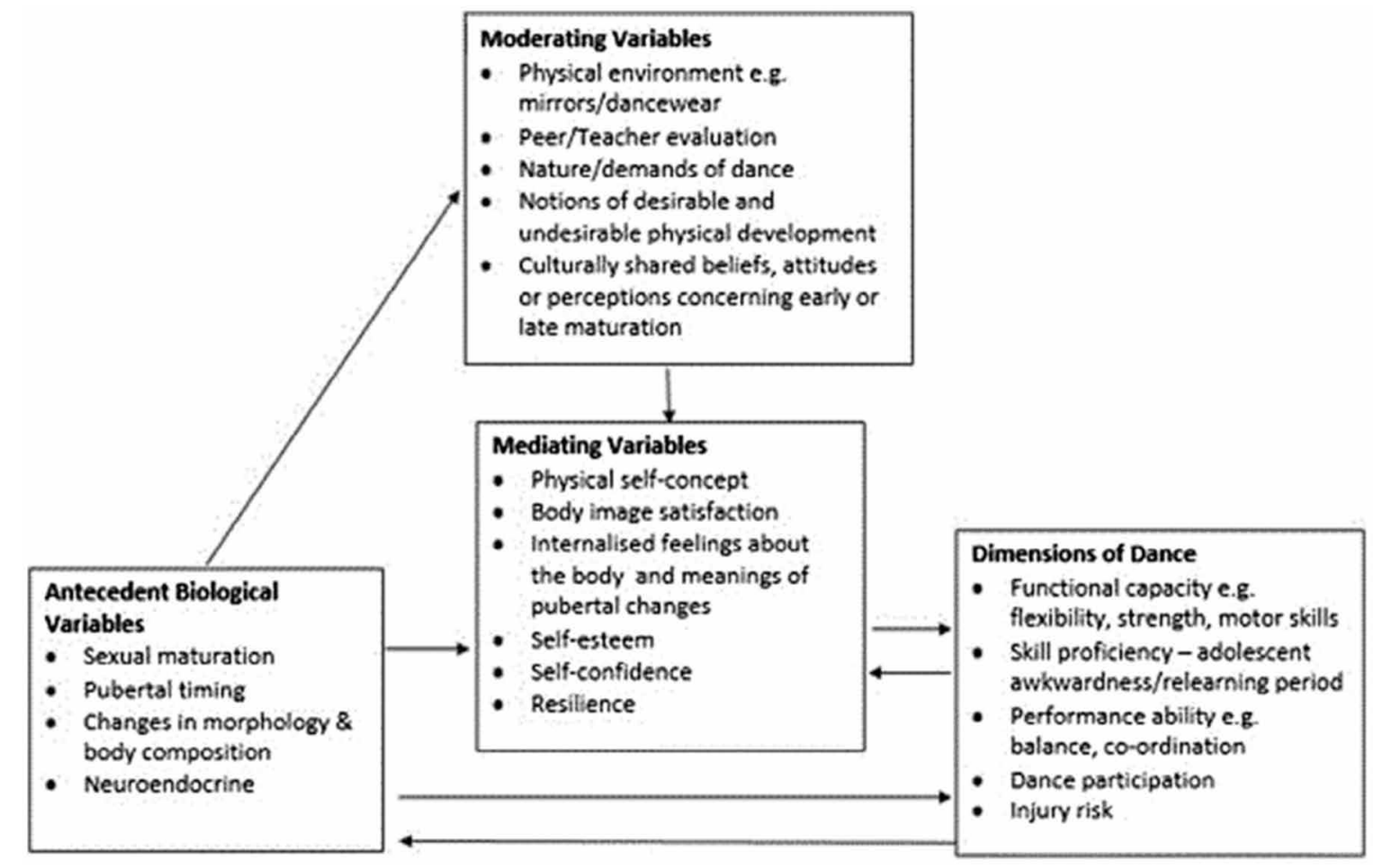

\section{NEGOTIATING THE DEVELOPMENTAL TASKS OF THE ADOLESCENT DANCER}

\section{Socio-Psychological Tasks}

Changes in pubertal status represent a dramatic change for the individual associated with both detrimental and beneficial outcomes. Changes throughout adolescence are characterised by rapid increases in both physical and mental capabilities: improvements in physical capacities, such as reaction time, immune function, resistance to injury and physical stress; and improvements in mental competencies, such as reasoning abilities (Dahl, 2004). While adolescence represents a period of strength and resilience, Dahl (2004) calls attention to the health paradox associated with this period; despite maturational improvements across several domains, overall morbidity and mortality rates increase by $200 \%$ during adolescence (Dahl, 2004). The primary sources associated with these rates are difficulties in controlling behaviour and emotion, resulting in concerns such as depression, eating disorders, alcohol and substance abuse (Dahl, 2004; Dahl \& Gunnar, 2009).

Individual adaptation to biological, psychological and physical changes at adolescence are significant in determining the direction of these outcomes for both males and females. For young dancers, this adaptation is situated within a highly complex setting and is influenced by factors including the environment, personal feelings about changes and the perception of changes by significant others (Cumming et al., 2012; Summers-Effler, 2004). In ballet, training exists in parallel with the years in which exploration 
and identity development are crucial (Lavallee \& Robinson, 2007). Increased individual consideration of who one is and where one belongs is a critical psychosocial development during adolescence (Crocetti, 2017). During this time, adolescent dancers are likely to go through the processes of questioning whether dance remains congruent with their shifting identity and self-view; thus, dancers may develop a discord between their identity and their dance participation.

Coupled with this identity shift are changes in self-esteem, with evidence to show that both males and females who develop a stronger identity in adolescence also demonstrate higher self-esteem (Marcia, 2017). One protective factor may be the social support offered by peers, whereby adolescents work through understanding how to relate to others, how to communicate optimally and how to control emotions in the face of changes in others. However, in dance, adolescents may be likely to perceive peers as competition, so students must be learning in an environment where cooperation and compassion are valued, known as a task-involving environment, over competition and criticism, known as an ego-involving environment (Nordin-Bates et al., 2014; Stark \& Newton, 2014).

Adolescence is proposed to be a time of confusion and crisis with regards to identity. Many young people temporarily adopt new beliefs, change their education or career goals, and physically change their appearance. Thus an individual is at increased risk of dropout from previously loved extracurricular activities such as dance. Females, in particular, are more likely to drop out from physical activity than male (Dumith et al., 2014; Fawkner et al., 2014). Several characteristics predict continued engagement with dance, including enjoyment, development of social relationships and being recognised as 'talented' (Aujla et al., 2014). Changes in social groups, finding a first romantic partner, and a lack of time to socialise with peers outside of a hobby may encourage dropout.

The age at which children begin ballet places them in a vulnerable position concerning internalising beliefs about the body and attractiveness (Petersen et al., 1980), which may be partially derived from the teacher and broader ballet culture (Pickard, 2013; Mitchell et al., 2016). In a vocational setting, the influence of the teacher and ballet context will be amplified, as interaction with the teacher is intensified and access to cultural contexts wider than the ballet school is limited (Buckroyd, 2000; Pickard, 2013). Moreover, the reference points and norms available to the individual will be based on what is expected in the ballet world rather than the broader social world; internalisation of these norms and building the self and identity around such specific body expectations may lead to a more negative perception of physical developments (Pickard, 2013).

The social-psychological climate is created by parents, peers, and teachers (Smith et al., 2007). Adolescence is commonly marked by increased independence from parents. However, participation in elite training is likely to require an adolescent to depend on their parents more so than their average peers, because parents are crucial in providing resources such as lifts to classes and competitions and financial support. Parents may form a means of crucial support and support the investment in dance training (Sanchez et al., 2012). Although the increased dependence on a parent may conflict with the desire for adolescent independence, research shows that female adolescents, in general, recognise the support provided by their parents, citing their mother, followed by their father, as the most significant source of support for their training (Risner, 2009).

For the dance student, the relationship with the dance teacher may impact heavily on development; the teacher plays a crucial role in the nurture of values and skills (Pummell \& Lavallee, 2009). In the ballet environment, in particular, individual development occurs within an environment whereby the pedagogical model often remains one of traditional authoritarianism, and in particular, females may be exposed to a particularly suppressive environment (Clements \& Nordin Bates 2020). Pickard (2012) 
notes that this can create binary ideas of 'right and wrong'. The teacher is often seen as the holder of knowledge of the codified technique and subsequent power, while the student is encouraged to be the passive recipient (Alterowitz 2014; Barr \& Oliver 2016). This environment may be at odds with the development of autonomy and critical thinking (Morris, 2003), vital in the transition to adulthood.

Considerable research has demonstrated the importance of autonomy for healthy motivation in dance (e.g. Hancox et al., 2017). In particular, several studies have shown that when training in an autonomysupportive environment, dancers are more likely to experience intrinsic motivation, that is, where the motivation to dance comes from an internalised enjoyment of dance, rather than external sources such as parental pressures or social/tangible rewards for participating (e.g. Quested \& Duda, 2011). An autonomysupportive environment in dance is one where the teacher provides a meaningful rationale for choices he or she makes, uses non-controlling, non-threatening language and offers choice and individuality for students (Deci \& Ryan, 1987). Many studies have shown the importance of autonomy-support for increasing student engagement in participation in the classroom and extracurricular activities (e.g. Black \& Deci, 2000; Gagne, 2003). In turn, autonomy-support is likely to increase a dancer's perception that their basic psychological needs are satisfied (Ryan \& Deci, 2001). Evidence shows that autonomy-support is likely to support adolescent dancers' creativity (Nordin-Bates, 2020; Clements \& Nordin-Bates, 2020).

Cultural and social factors external to the individual, such as the dance teacher, are essential moderators of individual adaptations to pubertal change (Mitchell et al., 2017). A young dancer's immediate social context may include objects of the physical environment, such as mirrors and dancewear, the studio's atmosphere, the dance teacher and peers (Mitchell et al., 2017). The dance teacher and other involved adults established the environment or "culture" of a specific dance studio. The environment created by the dance teacher is influenced by factors such as culturally shared beliefs, attitudes or perceptions concerning puberty per se and early or late maturation; cultural standards of attractiveness; and notions of desirable and undesirable physical development (Mitchell et al., 2017; Petersen \& Taylor, 1980). For example, in the social context of ballet, where later maturation is the norm for females, attitudes may be favourable towards later maturation due to the likelihood of maintaining a slimmer and more suitable physique (Brooks-Gunn \& Warren, 1985, Mitchell et al., 2016). These moderating factors are generally overlooked and are especially lacking in the context of dance, where focus on performance and evaluation makes moderating factors particularly important (Mitchell et al., 2017; Mitchell et al., 2016).

Identity development is a crucial task of adolescence, with social and peer context playing a significant role (McAdams \& Olson, 2010). Comparison to peers aids individuals in making sense of who they are and how they fit in the world (Adams \& Marshall, 1996). There is evidence of increased self-comparison with peers during adolescence. For example, it has been shown that females have heightened sensitivity to others' reactions to successes and failures than males (Murberg \& Bru, 2004), an experience probably heightened by the dominance of evaluation and assessment in classical dance training. Further still, females demonstrate increased desires to meet others' expectations (Wiklund et al., 2010).

Pickard's research $(2009,2012,2013,2015)$ on the lived experiences of young ballet dancers across four years emphasises the significance of developing within the social world of ballet or ballet sub-culture. Pickard notes how the identity of young ballet dancers is shaped by the need to attach positive meaning to lived experiences related to pain and suffering and learn to re-frame or suppress pain and negative emotions, accepted as part of the process of embodying an identity as a ballet dancer (Pickard, 2012; Pickard \& Bailey, 2009). In the ballet world, physical and emotional pain are understood as capital and currency that is traded to succeed, with dominant ideas, beliefs, norms, behaviours, values, and expectations of ballet culture transmitted via the ballet teacher (Pickard, 2012). For example, Megan (11 years 
old) stated: "I am so lucky because I am naturally slim and little, and that is exactly how you must be in ballet. I do not have to diet at the moment, but I know lots of my friends have to be very aware of what they eat because they have those sorts of bodies" (Pickard, 2013, p. 14). Megan's testimony is an example of how Megan has been shaped through her development within the ballet world. Megan has increased physical capital because of her naturally slim and petite physique. At the age of 11, Megan is already very aware of the notion of the ideal body for ballet and how important it is to succeed in the ballet world.

The relationship between the notion of an ideal body and identity as a ballet dancer is also important: "...if the body does not fit with the expectations of slim in size and shape then, they will not fit the expectations of ballet and will not be able to call themselves or claim identity as a ballet dancer" (Pickard, 2013, p. 16). Consistent with Mitchell et al. (2016), rejection of the body is described as a potential result of puberty for female dancers, and this type of rejection and disappointment is viewed by young dancers as an expected part of becoming a ballet dancer (Pickard, 2013). Dancers understand from a young age the notion of physical capital and the value of the body as a 'process of construction', an 'aesthetic project' and a 'product in performance' (Pickard, 2012; Pickard, 2013). Notably, the dancers studied in Pickard's research were involved in non-residential ballet training, meaning that they inhabit several different social worlds in addition to ballet, such as home or school. Pickard's explanation for why the influence of the social world of ballet appears to be dominant is related to the individual's commitment to becoming a ballet dancer and the development of one identity as a dancer (Pickard, 2012).

Pickard's work lends further support to the narrative of conflict between ballet and the adolescent body. Functional changes are highlighted as a challenge for dancers at puberty who are expected to remain 'co-ordinated', 'slim' and 'graceful' despite the changes they are experiencing (Buckroyd, 2000; Pickard, 2012): "I am really struggling at the moment because I have had a bizarre growth spurt and I am completely off balance. I hate that I cannot control my body at the moment. It is embarrassing. I keep looking at myself in the mirror in my leotard. I am trying everything to get it all back but I feel so clumsy" (Tracey, 15 years old) (Pickard, 2012, p. 33). This description demonstrates the conflict between the developing adolescent body and the performing body; while many young people experience selfconsciousness during adolescence, young dancers find themselves in a position where their developing body is constantly seen, in mirrors, by teachers and peers, in minimal clothing, and with permission to touch the body rarely sought by the teacher (Pickard, 2012).

\section{Cognitive Tasks}

While the norms and expectations of the ballet subculture prescribe delayed physical puberty, cultural constructions relating to cognitive and emotional development are more varied. At the same time, young dancers may be exposed to early opportunities to develop independence and self-reliance. Young dancers who undertake vocational training do so residentially, gaining responsibility for tasks such as laundry, organisation of schoolwork tasks and cooking, far in advance of their non-dance counterparts. These tasks offer early opportunities for cognitive and psychosocial development, which typically occur later in adolescence, and may also apply to those in recreational training, with the young person taking responsibility for practice and attendance of dance classes (Fraser-Thomas et al., 2005). Contrastingly, they may be restricted, for example, in their exposure to other cognitive-developmental opportunities. Young dancers, who often remain prepubescent in appearance, continue to be referred to and, in turn, may think of themselves as 'boys and girls' throughout much of their career (Buckroyd, 2000; Fay, 1997; Mitchell et al., 2016). 
During adolescence, a young person enters a period of formal-operational thought, demonstrating awareness and ability to think about abstract thought and hypothetical or metaphorical thinking or situations (Piaget \& Inhelder, 2000; Rafetseder et al., 2013) and enhanced moral development (Chiasson et al., 2017), but this comes hand in hand with increased egocentrism (Elkind, 1967; Frankenberger, 2000). These cognitive changes continue to be fine-tuned throughout adolescence, and recent evidence shows that the formation of new neural connections is ongoing long into an individual's twenties. Connections continue to develop in the prefrontal cortex, an area of the brain responsible for the organisation, strategic thinking and impulse control (Kayser et al., 2016). This neuroplasticity supports why adolescents are often unable to understand the consequences of their actions and perhaps why adolescents do not process information and think the same way an adult does.

During the adolescent period, a fundamental change observed is in heightened awareness of one's own body. This change is often accompanied by increased bodily dissatisfaction (e.g. Rosenblum \& Lewis, 1999). For adolescent dance students, the body is often exposed due to tight clothing, and there is increased time spent observing the body due to the prevalence of mirrors. Adolescents experience heightened self-consciousness, imagining how others might view their appearance. Alongside this, they are at a time of increased egocentrism, believing that others may view them as more important and are subsequently unable to differentiate between the feelings of themselves and others.

The cognitive, physical and social changes experienced by an individual during adolescence can affect the development of self-concept (Brinthaupt \& Lipka, 2002; Lerner \& Karabenick, 1974). Self-concept is described as how one perceives oneself and is developed through two primary sources, direct appraisals and reflected appraisals (Sebastian et al., 2008). Direct appraisals are based on an individual's reaction to past experiences and events, while reflected appraisals are based on their beliefs about how others see them (Sebastian et al., 2008). These two sources are increasingly important to the individual during adolescence when self and social comparison become more influential; individuals begin to compare their performance to their past and peers (Brinthaupt \& Lipka, 2002; Sebastian et al., 2008). Cultural expectations shape factors such as physical self-concept and internalised thoughts and feelings about the body and social contexts, and thus cultural norms and expectations around events such as puberty are integral to adolescent development (Mitchell et al., 2017; Pickard, 2013). For example, typical aspects of physical development, including breast development and widening of the hips, are likely to be less normalised in subcultures such as ballet and may be perceived more negatively by young dancers.

A further area for concern during adolescence is the anxiety that dancers may experience around performance, commonly referred to as performance anxiety. This anxiety is commonly understood as a combination of cognitive (e.g. worry, fear of failure, increased forgetfulness) and somatic (e.g. sweating, racing heart, muscular instability) symptoms, brought on by an impending evaluation (Osborne \& Franklin, 2002). Adolescence is a time when anxieties peak, mainly when assessments or evaluations are of a social nature, such as being assessed by teachers or peers or being observed (Erath, Flanagan \& Bierman, 2007). This proves a challenge for adolescent dancers, who are likely to be undertaking regular examinations, auditions and performances. Even participation in dance classes may increase adolescent anxieties. Research within dance has demonstrated that up to $35 \%$ of training and performing dancers experience regular performance anxiety; however, this number may be considerably higher in reality (Gaines, 2017). Little research specifically exploring adolescent dancers' performance anxiety has been conducted (Walker \& Nordin-Bates, 2010), although research in music demonstrates that around a third of gifted adolescents are handicapped by cognitive performance anxiety (Fehm \& Schmidt, 2006). 
Recent research has shown that adolescent dancers who are training full time at a vocational dance school experience higher levels of cognitive anxiety than somatic anxiety (Clegg \& Clements, in preparation). This is in line with the suggestion that cognitive concerns peak during adolescence. Qualitative evidence from the same authors provided an abundance of socially driven cognitive anxieties, such as perceived pressure to impress teachers and parents, as well as fear of competition from peers (Clements \& Clegg, in preparation). It is evident that expecting an adolescent to perform weekly is likely to cause experiences of cognitive anxiety, and the exposure to competition and direct comparison of dance students will also likely exacerbate self-comparison and self-critical evaluations, leaving adolescent dancers at risk of developing unhealthy perfectionistic tendencies (Stoeber, 2014).

\section{Physical Tasks}

The process of becoming mature, known as maturation, describes the development of three main biological systems: sexual, skeletal and somatic (Malina, 2014). With each biological system, maturity varies; sexual maturity refers to full functional reproductive capability; skeletal maturity, a fully ossified adult skeleton; and for somatic maturity where there is no definitive end-point which is defined as 'adult', maturity is assumed, for example, when growth in height is less than one centimetre per year (Malina et al., 2004; Ulijaszek et al., 1998).

Puberty is related to the process of sexual maturation and presents both opportunity and challenge for young dancers. Advantages such as increased strength and higher cognitive functioning occur in tandem with challenges such as gains in fat mass and disruption to flexibility, which may place individuals at increased risk within a dance training context (Dahl, 2004; Malina et al., 2004). The degree to which pubertal change positively or negatively impacts health is determined by numerous extrinsic and intrinsic factors (Petersen \& Taylor, 1980). These include physical changes of puberty (including the extent and order of pubertal changes); the social context of puberty; the social stimulus value of these changes within the context; the timing of maturation; and the individual's physical self-concept (e.g. Cumming et al., 2012; Petersen \& Taylor, 1980).

Physical developments, including changes in body size, shape and functioning resulting from hormonal changes, will change the physique to adult proportions and functions (Sugar, 1993). Early adolescence is characterised by biologically-based physical changes resulting from the onset of puberty. Puberty involves three endocrine events: andrenarche, gonadarche and activation of the growth axis (Blakemore et al., 2010; Petersen \& Taylor, 1980). Andrenarche, also described as the activation of the hypothalamic-pituitaryadrenal (HPA) axis, often begins before gonadarche, outside of the context of adolescence (Blakemore et al., 2010; Malina et al., 2004). This process contributes to developing secondary sex characteristics (Blakemore et al., 2010; Malina et al., 2004). Gonadarche is the biological process that occurs between age eight and fourteen in females (average age of eleven) and between nine and fifteen in males (average age of twelve), leading to activation of the HPA axis and resulting in reproductive maturity (Blakemore et al., 2010; Malina et al., 2004). Around age twelve for female and age fourteen for male, activation of the growth axis occurs, resulting in a linear growth spurt and changes in body size and composition (Blakemore et al., 2010; Marshall \& Tanner, 1969).

The biological basis of changes seen during pre-puberty and at puberty is evident and is both overt (e.g. breast development) and discrete (e.g. menarche). These hormonal changes directly affect the appearance and function of the body at puberty and beyond. The most pertinent and typically overt pubertal changes include increasing body mass, rapid changes in limb length, body size and distribution of body 
fat, and additionally for females, breast development, and the onset of menarche (Sugar, 1993; SummersEffler, 2004). The average sequence of pubertal change for females begins with breast development (as early as seven to eight years old), development of pubic hair (from age eleven), peak height velocity, i.e. the point of most rapid growth (average age twelve). For males, the earliest outward manifestation of puberty is the height spurt, starting between age ten and sixteen with average peak height velocity around age fourteen, and voice breaking around age fourteen to fifteen (Baker et al., 2012; McDowell et al., 2007). Although the onset of puberty typically occurs around eleven years of age for females and age twelve for males, individual variation is considerable, with some individuals experiencing puberty well in advance or delayed to same-age peers (Mendle, 2014).

For young dancers, the physical changes of puberty may have a significant impact. Changes to basic physical parameters (e.g. increases in limb length and changes in body proportions) can directly affect performance, technical ability, and injury risk (Bowerman et al., 2014; Daniels et al., 2001; Malina et al., 2004; Wyatt, 2015). Fundamental physical changes such as increases in height, fat and lean mass can affect aspects of physical performance, including strength, flexibility, muscular endurance and basic motor tasks, such as balance (Malina et al., 2004).

Most changes to physical performance are attributed to growth spurts and/or anatomical and functional changes in the joints during adolescence (Malina et al., 2004). For young dancers, these changes are likely to affect performance in numerous ways, including decreases in overall technical skill, control, strength, flexibility, coordination and balance, and changes to alignment necessitated by increased limb length relative to the spine (Bowerman et al., 2015; Daniels et al., 2001; Mitchell et al., 2016). These changes will impact core dance movements; for example, reduced strength and flexibility result in lower leg extensions, while reduced balance and coordination affect the pirouettes. As technical control decreases, injury risk increases (Daniels et al., 2001).

Bowerman and colleagues (2015) noted the additional challenge and increased injury risk associated with adapting to pubertal changes, the 'relearning period'. During this period, young dancers must relearn technique and re-programme this technique to adjust to new biomechanical challenges, such as decreased strength, power and flexibility, and rapid limb length changes (Bowerman et al., 2015; Phillips, 1999). The increased susceptibility to injury associated with the adolescent growth spurt through factors such as transient low bone mass and adjustment to new biomechanical challenges often coincides with increased intensity of dance training. While researchers have recommended monitoring the intensity and volume of training to avoid overuse injuries during periods of rapid growth, no clear solution has been presented which addresses the significant differences between individuals in terms of the biological timing of maturation (Bowerman et al., 2015; Daniels et al., 2001; Horobeanu et al., 2017).

Maturation timing describes when specific maturational events occur, such as the onset of menarche in girls, and is usually classified as advanced (early), average (on time) or delayed (late) relative to a specific cohort (Malina et al., 2004). For example, current research reports 12.4 - 12.8 years as the average age of menarche (Baker et al., 2012; Malina et al., 2004; McDowell et al., 2007). Individuals who fall below one year of this age would be described as 'advanced' and those one year above as 'delayed' (Malina et al., 2004). Maturation tempo is defined by the rate at which an individual progresses through maturation (Malina et al., 2004). Maturation timing is largely genetically determined (e.g. Rowe, 2002; Treloar \& Martin, 1990) but influenced by environmental stressors (acute and chronic), which delay or accelerate the maturation process (Gluckman \& Hanson, 2006). Environmental stressors include nutritional, prenatal and postnatal influences. Involvement in intensive dance training may also constitute 
an environmental stressor, yet our understanding of the effect is limited due to difficulty distinguishing the independent effects of training from those of average growth (Baxter-Jones \& Maffulli, 2002). Research in gymnastics suggests that intensive training does not appear to attenuate pubertal growth and maturation rate, timing or tempo (Malina et al., 2013). Similar findings have been reported in novice dancers across various dance styles, though there is no longitudinal data from vocational ballet training (Matthews et al., 2006).

The biological timing of pubertal events can be pivotal in determining physical development trajectory; late maturation leads to a more desirable, lean physique and earlier maturation, presenting more significant aesthetic challenges for young dancers (Gay et al., 2014). Whether or not particular timing is favourable may depend on the type of activity in which a young person is involved. Early maturation can be seen as advantageous in physical activities which involve power and strength. In contrast, late maturation, which results in a more petite and more lean physique, may be more favourable for endurance, aesthetic sports or performing arts, such as ballet (e.g. Ackland, Elliott, \& Richards, 2003).

Existing literature has only explored maturation timing in female, as yet no comparable data is available for male. For females in ballet, earlier menarche and greater breast development have been highlighted in those who did not complete professional training, suggesting that certain physical developments are not considered conducive to a ballet career (Hamilton et al., 1997). While early literature indicates a preference for later maturation in ballet and other dance styles (e.g. Hamilton et al., 1988; Hamilton et al., 1997; Steinberg et al., 2008; Warren, 1989), recent research questions long-standing assumptions about late maturation as purely advantageous for young ballet dancers. Research with dance teachers suggesting that early maturation is preferable in terms of 'getting the growing out of the way' before more serious training commences (Mitchell et al., 2016; Mitchell et al., 2020).

Literature is essentially in agreement that early maturation is not socially advantageous for females, particularly within social contexts such as ballet, where there are clear preferences and benefits associated with later maturation (Burckhardt et al., 2011; Hamilton et al., 1988; Hamilton et al., 1997; Pickard, 2012, 2013; Steinberg et al., 2008; Warren, 1989). However, research conducted with ten ballet teachers working with vocational and recreational dancers reported mixed opinions regarding the benefits associated with early, average and late maturation (Brooks-Gunn \& Warren, 1985; Mitchell et al., 2016). There were mixed opinions of what might be most advantageous for a young dancer in terms of awareness of the impacts of different timing within the dance context. Opinions ranged from views in agreement with previous findings that later maturation gives a nicer 'look'; to perceiving earlier maturation to be a potential advantage in terms of getting a lot of the 'growing done' before serious training begins; to view the timing of maturation as irrelevant (Mitchell et al., 2016). Teachers described advantages and disadvantages relative to when the testing of a young dancer occurs. For example, it might be advantageous to mature later in auditioning for schools as it provides a better 'look'; though equally it might be advantageous to mature earlier, to be ready for when more intensive training begins (Mitchell et al., 2016). These findings diverge from the consensus presented in the existing literature that up to $70 \%$ of professional ballet dancers are late maturing (Hamilton et al., 1988; Hamilton et al., 1985). While there are, in many ways, advantages associated with later maturation in the ballet world, there are also potential merits to earlier maturation that have not yet been explored. In addition, it is essential to acknowledge that existing literature reporting a bias toward late maturation may be outdated, and therefore the views of ballet teachers in this study may represent more current views (Mitchell et al., 2016). Further research is needed to establish current trends in ballet. 
Congruent with Mitchell et al., 2016 and 2020, research on injury risk highlights several challenges for the late-maturing dancer. The timing of maturation may have particular implications for injury risk in young female dancers who are delayed in maturation. In those with delayed maturation, fractures have been reported to rise with increasing age at menarche (Warren et al., 1986). This increased risk of injury is associated with prolonged hypoestrogenism, referring to a lower than average oestrogen level, and is a well-recognised complication of weight loss, dieting and physical training in female (Tanchev et al., 2000; Warren et al., 1986). Delayed growth and maturation lead to a prolongation of the vulnerable growing years, exposing the growth plates to the influence of adverse mechanical factors, such as pressure, impact and microtrauma, for a longer period (Tanchev et al., 2000). Further injury risk factors identified in adolescent gymnasts, which may apply to young dancers, including skeletal immaturity, insufficient rest periods and repetitive movements (DePalma, 2006; Wyatt, 2015).

The majority of research studies in this area show a bias towards later maturation in ballet, with differing explanations as to why and a lack of research in other dance styles (Burckhardt et al., 2011; Hamilton et al., 1988; Hamilton et al., 1997; Pickard, 2012, 2013; Steinberg et al., 2008; Warren, 1989). However, the most compelling conclusion suggests that the trend towards delayed maturation in ballet results from a combination of factors, such as maintaining a low body mass during adolescence, inadequate nutrition, low body fat, high ratio of lean mass to body mass in conjunction with a proper selection of these body types and self-selection of late maturers into ballet (Bowerman et al., 2015; Steinberg et al., 2008).

The timing of pubertal change appears to affect not only physical wellbeing and the ability to meet the social expectations of the ballet world but also on individuals' subsequent psychological wellbeing. The social context of ballet has been found to amplify the effects of maturation timing and the resulting detriment to psychosocial wellbeing (Brooks-Gunn \& Warren, 1985). Early and on-time maturation within a ballet training context was associated with higher incidences of eating pathologies than in early and on time non-dancers (Brooks-Gunn \& Warren, 1985). It was hypothesised that effects of maturation timing might be moderated by the social context and cultural and individual beliefs about the importance of behaviours associated with maturation (Brooks-Gunn \& Warren, 1985).

Overall, there are several physical and psychological challenges associated with developing in the context of ballet; however, it is also apparent that there may be many ways to mitigate or ease some of these challenges (Mitchell et al., 2017; Mitchell et al., 2016). It is important to consider approaches that directly address the healthy development of the young people within dance training.

\section{RECOMMENDATIONS AND CONCLUSION}

Puberty represents a challenging time for young dancers, but many challenges can be overcome with the dance teacher's guidance. Awareness of physical changes that dancers experience during puberty, gender differences, and how changes impact performance and injury risk can equip dance educators to facilitate this transition. Schools and teachers can reduce the risk of injury during adolescence by monitoring and measuring growth and maturation, which can help predict the most rapid periods of growth, identify maturity timing, and inform adjustments to training load. Remaining mindful of additional risk factors such as insufficient rest, reduced flexibility and strength, and individual differences in maturity timing can enable personalised approaches to young dancers' training. Similarly, parents should be aware of changes in the adolescent's behaviour and overt and covert displays of dissatisfaction with dance participation. 
While the physical changes of puberty present challenge to dancers in terms of technique, performance and injury risk, further conflicts can arise between pubertal changes and physical appearance, particularly when auditioning or being assessed. The desirability of pubertal changes is sex-dependent; in particular, the more overt female changes such as breast development and increases in fat mass, alongside temporary changes to physical capabilities, will likely affect self-consciousness, self-confidence, body image and identity. Healthy adaptation to physical changes is critical, as it can heavily influence the trajectory of future psychological wellbeing (Moore, 2016; Dahl, 2004). Healthy adaptation is often affected by social context, where involvement in elite sport or dance at adolescence may place considerably higher pressure on successful and rapid adaptation to physical changes.

Awareness and sensitivity to a dancer's stage of psychological development are critical (see guidance below on how to help young dancers to develop a positive sense of self). Understanding their ability to negotiate particular challenges relative to their stage of development can ensure the right amount of support and encouragement and management of expectation (Mitchell et al., 2017). For example, during early adolescence, in particular, young people need increased encouragement and motivation to sustain practice as they are less able to connect the importance of regular practice to the logical outcome of future dancing success and also less able to form a realistic understanding of the role dance may have in their lives in the future (Brown et al., 2017). In comparison, parents have become more independent of parental figures and rely on guidance from other significant adults; late adolescents will benefit from the dance teacher's support and guidance.

Teachers can work to optimise young dancers' development and psychological wellbeing by creating adaptive learning environments via positive teaching behaviours and both direct and indirect actions to make the pubertal transition less stressful.

1. Focus cues/comments on positive messages and how movements feel rather than what the body looks like

2. Raise awareness amongst dancers and parents about normal and temporary changes associated with maturation

3. Create a protective environment using direct and indirect actions (e.g. guiding dancer aspirations toward appropriate dance pathways).

\section{Guidance for Helping Young Dancers to Develop a Positive Sense of Self}

To help young dancers to develop a positive sense of self, dance teachers can:

- Avoid using comments that compare students instead of creating a positive task-involving motivational climate, supporting students' basic psychological needs.

- Use language which encourages personal growth and development, providing options that recognise that skill development will be different for class members.

- $\quad$ Reduce use of/focus on the mirror to help students to minimise comparison with others

- Be flexible about uniform, particularly where young dancers have matured in advance of peers and adjust to a changing body.

- Focus time/attention towards aspects other than technique (which may progress more slowly during this time), such as musicality, performance and strengthening. 
In summary, the teacher has an essential role in facilitating a positive and adaptive transition through puberty. Young dancers place a high value on teachers' comments and opinions of the teacher, and these views can be influential in determining how a young dancer perceives and adjusts to pubertal changes.

\section{ACKNOWLEDGMENT}

We want to thank the reviewers of this chapter: Ms Maria Cristina Lopes, Ms Marcela Delabary, Dr Camila Bicalho and Dr Bárbara Pessali-Marques, for their effort and expertise.

\section{REFERENCES}

Ackland, T., Elliott, B., \& Richards, J. (2003). Gymnastics: Growth in body size affects rotational performance in women's gymnastics. Sports Biomechanics, 2(2), 163-176. doi:10.1080/14763140308522815 PMID:14737925

Adams, G. R., \& Marshall, S. K. (1996). A developmental social psychology of identity: Understanding the person-in-context. Journal of Adolescence, 19(5), 429-442. doi:10.1006/jado.1996.0041 PMID:9245296

Alterowitz, G. (2014). Toward a feminist ballet pedagogy: Teaching strategies for ballet technique classes in the twenty-first century. Journal of Distance Education, 14(1), 8-17. doi:10.1080/15290824.2013. 824579

Anshel, M. H. (2004). Sources of disordered eating patterns between ballet dancers and non-dancers. Journal of Sport Behavior, 27(2), 115.

Arnett, J. J. (2010). Emerging adulthood(s). Bridg Cult Dev approaches to Psychol New Synth theory. Research Policy, 255-275.

Aujla, I. J., Nordin-Bates, S. M., \& Redding, E. (2015). Multidisciplinary predictors of adherence to contemporary dance training: Findings from the UK Centres for Advanced Training. Journal of Sports Sciences, 33(15), 1564-1573. doi:10.1080/02640414.2014.996183 PMID:25554823

Baker, J. H., Thornton, L. M., Bulik, C. M., Kendler, K. S., \& Lichtenstein, P. (2012). Shared genetic effects between age at menarche and disordered eating. The Journal of Adolescent Health, 51(5), 491-496. doi:10.1016/j.jadohealth.2012.02.013 PMID:23084171

Barr, S., \& Oliver, W. (2016). Feminist pedagogy, body image, and the dance technique class. Research in Dance Education, 17(2), 97-112. doi:10.1080/14647893.2016.1177008

Baxter-Jones, A. D. G., \& Maffulli, N. (2002). Intensive training in elite young female athletes. British Journal of Sports Medicine, 36(1), 13-15. doi:10.1136/bjsm.36.1.13 PMID:11867484

Benn, T., \& Walters, D. (2001). Between Scylla and Charybdis. Nutritional education versus body culture and the ballet aesthetic: The effects on the lives of female dancers. Research in Dance Education, 2(2), 139-154. doi:10.1080/14647890120100773 
Beunen, G., Ostyn, M., Renson, R., Simons, J., \& Van Gerven, D. (1976). Skeletal maturation and physical fitness of girls aged 12 through 16. Hermes, 10, 445-457.

Black, A. E., \& Deci, E. L. (2000). The effects of instructors' autonomy support and students' autonomous motivation on learning organic chemistry: A self-determination theory perspective. Science Education, 84(6), 740-756. doi:10.1002/1098-237X(200011)84:6<740::AID-SCE4>3.0.CO;2-3

Blakemore, S. J., Burnett, S., \& Dahl, R. E. (2010). The role of puberty in the developing adolescent brain. Human Brain Mapping, 31(6), 926-933. doi:10.1002/hbm.21052 PMID:20496383

Bor, W., Dean, A. J., Najman, J., \& Hayatbakhsh, R. (2014). Are child and adolescent mental health problems increasing in the 21st century? A systematic review. The Australian and New Zealand Journal of Psychiatry, 48(7), 606-616. doi:10.1177/0004867414533834 PMID:24829198

Bowerman, E., Whatman, C., Harris, N., Bradshaw, E., \& Karin, J. (2014). Are maturation, growth and lower extremity alignment associated with overuse injury in elite adolescent ballet dancers? Physical Therapy in Sport, 15(4), 234-241. doi:10.1016/j.ptsp.2013.12.014 PMID:24726033

Bowerman, E. A., Whatman, C., Harris, N., \& Bradshaw, E. (2015). A Review of the Risk Factors for Lower Extremity Overuse Injuries in Young Elite Female Ballet Dancers. Journal of Dance Medicine \& Science: Official Publication of the International Association for Dance Medicine \& Science, 19(2), 51-56. doi:10.12678/1089-313X.19.2.51 PMID:26045395

Brinthaupt, T. M., \& Lipka, R. P. (2002). Understanding early adolescent self and identity: An introduction. Understanding early adolescent self and identity: Applications and Interventions, 1-21.

Brooks-Gunn, J., Attie, I., Burrow, C., Rosso, J. T., \& Warren, M. P. (1989). The impact of puberty on body and eating concerns in athletic and nonathletic contexts. The Journal of Early Adolescence, 9(3), 269-290. doi:10.1177/0272431689093006

Brooks-Gunn, J., \& Warren, M. (1985). The effects of delayed menarche in different contexts: Dance and non-dance students. Journal of Youth and Adolescence, 14(4), 285-300. doi:10.1007/BF02089235 PMID:24301222

Brown, K. A., Patel, D. R., \& Darmawan, D. (2017). Participation in sports in relation to adolescent growth and development. Translational Pediatrics, 6(3), 150-159. doi:10.21037/tp.2017.04.03 PMID:28795005

Buckroyd, J. (Ed.). (2000). The student dancer: Emotional aspects of the teaching and learning of dance. Princeton Book Company Pub.

Burckhardt, P., Wynn, E., Krieg, M.-A., Bagutti, C., \& Faouzi, M. (2011). The effects of nutrition, puberty and dancing on bone density in adolescent ballet dancers. Journal of Dance Medicine \& Science: Official Publication of the International Association for Dance Medicine \& Science, 15(2), 51-61. PMID:21703093

Carr, S., \& Wyon, M. (2003). The impact of motivational climate on dance students' achievement goals, trait anxiety, and perfectionism. Journal of Dance Medicine \& Science: Official Publication of the International Association for Dance Medicine \& Science, 7(4), 105-114. 
Chiasson, V., Vera-Estay, E., Lalonde, G., Dooley, J. J., \& Beauchamp, M. H. (2017). Assessing social cognition: Age-related changes in moral reasoning in childhood and adolescence. The Clinical Neuropsychologist, 31(3), 515-530. doi:10.1080/13854046.2016.1268650 PMID:28080301

Clegg, H., Owton, H., \& Allen-Collinson, J. (2016). The cool stuff!: Gender, dance and masculinity. Psychology of Women Section Review, 18(2), 6-16.

Clegg, H., Owton, H., \& Allen-Collinson, J. (2019). Attracting and Retaining Boys in Ballet. Journal of Distance Education, 19(4), 158-167. doi:10.1080/15290824.2018.1472381

Clements, L., \& Nordin-Bates, S. M. (2020). Inspired or Inhibited? Choreographers' Views on How Classical Ballet Training Shaped Their Creativity. Journal of Dance Education, 1-12.

Crane, J., \& Temple, V. (2015). A systematic review of dropout from organised sport among children and youth. European Physical Education Review, 21(1), 114-131. doi:10.1177/1356336X14555294

Crocetti, E. (2017). Identity formation in adolescence: The dynamic of forming and consolidating identity commitments. Child Development Perspectives, 11(2), 145-150. doi:10.1111/cdep.12226

Cumming, S. P., Sherar, L. B., Gammon, C., Standage, M., \& Malina, R. M. (2012). Physical activity and physical self-concept in adolescence: A comparison of girls at the extremes of the biological maturation continuum. Journal of Research on Adolescence, 22(4), 746-757. doi:10.1111/j.1532-7795.2012.00821.x

Dahl, R. (2004). Adolescent brain development: A period of vulnerabilities and opportunities - Keynote address. Adolescent Brain Development: Vulnerabilities and Opportunities, 1021(1), 1-22. doi:10.1196/ annals.1308.001 PMID:15251869

Dahl, R. E., \& Gunnar, M. R. (2009). Heightened stress responsiveness and emotional reactivity during pubertal maturation: Implications for psychopathology. Development and Psychopathology, 21(1), 1-6. doi:10.1017/S0954579409000017 PMID:19144219

Daniels, K., Rist, R., \& Rijven, M.The Education Committee of the Inte. (2001). The Challenge of the Adolescent Dancer. Journal of Distance Education, 1(2), 74-76. doi:10.1080/15290824.2001.10387180

Deci, E. L., \& Ryan, R. M. (1987). The support of autonomy and the control of behavior. Journal of Personality and Social Psychology, 53(6), 1024-1037. doi:10.1037/0022-3514.53.6.1024PMID:3320334

DePalma, M. J., \& Bhargava, A. (2006). Nonspondylolytic etiologies of lumbar pain in the young athlete. Current Sports Medicine Reports, 5(1), 44-50. doi:10.1097/01.CSMR.0000306518.32796.fd PMID:16483516

Dumith, S. C., Gigante, D. P., Domingues, M. R., \& Kohl, H. W. III. (2011). Physical activity change during adolescence: A systematic review and a pooled analysis. International Journal of Epidemiology, 40(3), 685-698. doi:10.1093/ije/dyq272 PMID:21245072

Elkind, D. (1967). Egocentrism in adolescence. Child Development, 38(4), 1025-1034. doi:10.2307/1127100 PMID:5583052 
Erath, S. A., Flanagan, K. S., \& Bierman, K. L. (2007). Social anxiety and peer relations in early adolescence: Behavioral and cognitive factors. Journal of Abnormal Child Psychology, 35(3), 405-416. doi:10.100710802-007-9099-2 PMID:17265192

Fawkner, S., Henretty, J., Knowles, A.-M., Nevill, A., \& Niven, A. (2014). The influence of maturation, body size and physical self-perceptions on longitudinal changes in physical activity in adolescent girls. Journal of Sports Sciences, 32(4), 392-401. doi:10.1080/02640414.2013.825733 PMID:24015894

Fay, M. (1997). Mind over Body: The development of the dancer - the role of the teacher. A \& C Black.

Fehm, L., \& Schmidt, K. (2006). Performance anxiety in gifted adolescent musicians. Journal of Anxiety Disorders, 20(1), 98-109. doi:10.1016/j.janxdis.2004.11.011 PMID:16325117

Frankenberger, K. D. (2000). Adolescent egocentrism: A comparison among adolescents and adults. Journal of Adolescence, 23(3), 343-354. doi:10.1006/jado.2000.0319 PMID:10837112

Fraser-Thomas, J., Côté, J., \& Deakin, J. (2005). Youth sport programs: An avenue to foster positive youth development. Physical Education and Sport Pedagogy, 10(1), 19-40. doi:10.1080/1740898042000334890

Fredricks, J. A., \& Eccles, J. S. (2008). Participation in extracurricular activities in the middle school years: Are there developmental benefits for African American and European American youth? Journal of Youth and Adolescence, 37(9), 1029-1043. doi:10.100710964-008-9309-4

Gagne, M. (2003). Autonomy support and need satisfaction in the motivation and wellbeing of gymnasts. Journal of Applied Sport Psychology, 15(4), 372-390. doi:10.1080/714044203

Gay, J. L., Monsma, E. V., Smith, A. L., DeFreese, J., \& Torres-McGehee, T. (2014). Assessment of growth and maturation in female athletes at a single point in time. Women in Sport and Physical Activity Journal, 22(2), 76-82. doi:10.1123/wspaj.2014-0018

Gluckman, P. D., \& Hanson, M. A. (2006). Evolution, development and timing of puberty. Trends in Endocrinology and Metabolism, 17(1), 7-12. doi:10.1016/j.tem.2005.11.006 PMID:16311040

Hamilton, L. H., Brooks-Gunn, J., \& Warren, M. P. (1985). Sociocultural influences on eating disorders in professional female ballet dancers. International Journal of Eating Disorders, 4(4), 465-477. doi:10.1002/1098-108X(198511)4:4<465::AID-EAT2260040407>3.0.CO;2-0

Hamilton, L. H., Brooks-Gunn, J., Warren, M. P., \& Hamilton, W. G. (1988). The role of selectivity in the pathogenesis of eating problems in ballet dancers. Medicine and Science in Sports and Exercise, 20(6), 560-565. doi:10.1249/00005768-198812000-00007 PMID:3070257

Hamilton, L. H., Hamilton, W. G., Warren, M. P., Keller, K., \& Molnar, M. (1997). Factors contributing to the attrition rate in elite ballet students. Journal of Dance Medicine \& Science: Official Publication of the International Association for Dance Medicine \& Science, 1(4), 131-139.

Hancox, J. E., Quested, E., Ntoumanis, N., \& Duda, J. L. (2017). Teacher-created social environment, basic psychological needs, and dancers' affective states during class: A diary study. Personality and Individual Differences, 115, 137-143. doi:10.1016/j.paid.2016.03.033 
Horobeanu, C., Jones, T., \& Johnson, A. (2017). Can we limit training days lost due to osgood schlatters disease in junior squash athletes? British Journal of Sports Medicine, 51(4), 331-332. doi:10.1136/ bjsports-2016-097372.122

Kayser, A. S., Op de Macks, Z., Dahl, R. E., \& Frank, M. J. (2016). A neural correlate of strategic exploration at the onset of adolescence. Journal of Cognitive Neuroscience, 28(2), 199-209. doi:10.1162/ jocn_a_00896 PMID:26488590

Lavallee, D., \& Robinson, H. K. (2007). In pursuit of an identity: A qualitative exploration of retirement from women's artistic gymnastics. Psychology of Sport and Exercise, 8(1), 119-141. doi:10.1016/j. psychsport.2006.05.003

Lerner, R. M., \& Karabenick, S. A. (1974). Physical attractiveness, body attitudes, and self-concept in late adolescents. Journal of Youth and Adolescence, 3(4), 307-316. doi:10.1007/BF02214744 PMID:24414371

Leversen, I., Danielsen, A. G., Birkeland, M. S., \& Samdal, O. (2012). Basic psychological need satisfaction in leisure activities and adolescents' life satisfaction. Journal of Youth and Adolescence, 41(12), 1588-1599. doi:10.100710964-012-9776-5 PMID:22627625

Malina, R. M., Bouchard, C., \& Bar-Or, O. (2004). Growth, maturation, and physical activity. Human kinetics. doi:10.5040/9781492596837

Marcia, J. E. (2017). Ego-Identity Status: Relationship to Change in Self-Esteem. Social Encounters: Contributions to Social Interaction, 340.

Marshall, W. A., \& Tanner, J. M. (1969). Variations in pattern of pubertal changes in girls. Archives of Disease in Childhood, 44(235), 291-303. doi:10.1136/adc.44.235.291 PMID:5785179

Matthews, B. L., Bennell, K. L., Mckay, H. A., Khan, K. M., Baxter-Jones, A. D., Mirwald, R. L., \& Wark, J. D. (2006). The influence of dance training on growth and maturation of young females: A mixed longitudinal study. Annals of Human Biology, 33(3), 342-356. doi:10.1080/03014460600635951 PMID: 17092871

McAdams, D. P., \& Olson, B. D. (2010). Personality development: Continuity and change over the life course. Annual Review of Psychology, 61(1), 517-542. doi:10.1146/annurev.psych.093008.100507 PMID:19534589

McDowell, M. A., Brody, D. J., \& Hughes, J. P. (2007). Has age at menarche changed? Results from the National Health and Nutrition Examination Survey (NHANES) 1999-2004. The Journal of Adolescent Health, 40(3), 227-231. doi:10.1016/j.jadohealth.2006.10.002 PMID:17321422

McEwen, K., \& Young, K. (2011). Ballet and pain: Reflections on a risk-dance culture. Qualitative Research in Sport, Exercise and Health, 3(2), 152-173. doi:10.1080/2159676X.2011.572181

Mendle, J. (2014). Beyond pubertal timing: New directions for studying individual differences in development. Current Directions in Psychological Science, 23(3), 215-219. doi:10.1177/0963721414530144

Mendle, J., Turkheimer, E., \& Emery, R. E. (2007). Detrimental psychological outcomes associated with early pubertal timing in adolescent girls. Developmental Review, 27(2), 151-171. doi:10.1016/j. dr.2006.11.001 PMID:20740062 
Mitchell, S., Haase, A., Cumming, S., \& Malina, R. M. (2017). Understanding growth and maturation in the context of ballet: A biocultural approach. Research in Dance Education, 18(3), 291-300. doi:10 $.1080 / 14647893.2017 .1387525$

Mitchell, S. B., Haase, A. M., \& Cumming, S. P. (2020). Experiences of delayed maturation in female vocational ballet students: An interpretative phenomenological analysis. Journal of Adolescence, 80, 233-241. doi:10.1016/j.adolescence.2020.03.005 PMID:32208172

Mitchell, S. B., Haase, A. M., \& Cumming, S. P. (in press). Experiences of on time maturation in adolescent ballet dancers. An Interpretative Phenomenological Analysis.

Mitchell, S. B., Haase, A. M., Malina, R. M., \& Cumming, S. P. (2016). The role of puberty in the making and breaking of young ballet dancers: Perspectives of dance teachers. Journal of Adolescence, 47, 81-89. doi:10.1016/j.adolescence.2015.12.007 PMID:26775190

Moore, S. R., McKone, K.M., \& Mendle, J. (2016). Recollections of puberty and disordered eating in young women. Journal of Adolescence, 53, 180-188. doi:10.1016/j.adolescence.2016.10.011 PMID:27814495

Morris, G. (2003). Problems with ballet: Steps, style and training. Research in Dance Education, 4(1), 17-30. doi:10.1080/14647890308308

Murberg, T. A., \& Bru, E. (2004). Social support, negative life events and emotional problems among Norwegian adolescents. School Psychology International, 25(4), 387-403. doi:10.1177/0143034304048775

Nordin-Bates, S. M. (2020). Striving for Perfection or for Creativity? A Dancer's Dilemma. Journal of Distance Education, 20(1), 23-34. doi:10.1080/15290824.2018.1546050

Nordin-Bates, S. M., Hill, A. P., Cumming, J., Aujla, I. J., \& Redding, E. (2014). A longitudinal examination of the relationship between perfectionism and motivational climate in dance. Journal of Sport \& Exercise Psychology, 36(4), 382-391. doi:10.1123/jsep.2013-0245 PMID:25226607

Nordin-Bates, S. M., Quested, E., Walker, I. J., \& Redding, E. (2012). Climate change in the dance studio: Findings from the UK centres for advanced training. Sport, Exercise, and Performance Psychology, l(1), 3-16. doi:10.1037/a0025316

Osborne, M. S., \& Franklin, J. (2002). Cognitive processes in music performance anxiety. Australian Journal of Psychology, 54(2), 86-93. doi:10.1080/00049530210001706543

Petersen, A. C., \& Taylor, B. (1980). The biological approach to adolescence: Biological change and psychological adaptation. In J. Adelson (Ed.). Handbook of Adolescent Psychology. Wiley.

Phillips, C. (1999). Strength training of dancers during the adolescent growth spurt. Journal of Dance Medicine \& Science: Official Publication of the International Association for Dance Medicine \& Science, 3(2), 66-72.

Piaget, J., \& Inhelder, B. (2000). The development of perception. Basic Book.

Pickard, A. (2012). Schooling the dancer: The evolution of an identity as a ballet dancer. Research in Dance Education, 13(1), 25-46. doi:10.1080/14647893.2011.651119 
Pickard, A. (2013). Ballet body belief: Perceptions of an ideal ballet body from young ballet dancers. Research in Dance Education, 14(1), 3-19. doi:10.1080/14647893.2012.712106

Pickard, A. (2015). Ballet body narratives: Pain, pleasure and perfection in embodied identity. Peter Lang. doi:10.3726/978-3-0353-0717-7

Pickard, A., \& Bailey, R. (2009). Crystallising experiences among young elite dancers. Sport Education and Society, 14(2), 165-181. doi:10.1080/13573320902809047

Pummell, B., \& Lavallee, D. (2009, June). Development of a model of junior-to-senior transition. Proceedings of the 12th World Congress of Sport Psychology.

Quested, E., \& Duda, J. L. (2009). Perceptions of the motivational climate, need satisfaction, and indices of well-and ill-being among hip hop dancers. Journal of Dance Medicine \& Science: Official Publication of the International Association for Dance Medicine \& Science, 13(1), 10-19. PMID:19416610

Quested, E., \& Duda, J. L. (2010). Exploring the social-environmental determinants of well-and illbeing in dancers: A test of basic needs theory. Journal of Sport \& Exercise Psychology, 32(1), 39-60. doi:10.1123/jsep.32.1.39 PMID:20167951

Quested, E., \& Duda, J. L. (2011). Perceived autonomy support, motivation regulations and the selfevaluative tendencies of student dancers. Journal of Dance Medicine \& Science: Official Publication of the International Association for Dance Medicine \& Science, 15(1), 3-14. PMID:21703088

Rafetseder, E., Schwitalla, M., \& Perner, J. (2013). Counterfactual reasoning: From childhood to adulthood. Journal of Experimental Child Psychology, 114(3), 389-404. doi:10.1016/j.jecp.2012.10.010 PMID:23219156

Risner, D. (2009) When Boys dance: Moving masculinities and cultural resistance in dance training and education. In Conference proceedings of Dance and the Child International (daCi), Kingston, Jamaica.

Rosenblum, G. D., \& Lewis, M. (1999). The relations among body image, physical attractiveness, and body mass in adolescence. Child Development, 70(1), 50-64. doi:10.1111/1467-8624.00005 PMID:10191514

Rowe, D. C. (2002). On genetic variation in menarche and age at first sexual intercourse: A critique of the Belsky-Draper hypothesis. Evolution and Human Behavior, 23(5), 365-372. doi:10.1016/S10905138(02)00102-2

Sanchez, E. N., Aujla, I. J., \& Nordin-Bates, S. (2013). Cultural background variables in dance talent development: Findings from the UK centres for advanced training. Research in Dance Education, 14(3), 260-278. doi:10.1080/14647893.2012.712510

Sebastian, C., Burnett, S., \& Blakemore, S. J. (2008). Development of the self-concept during adolescence. Trends in Cognitive Sciences, 12(11), 441-446. doi:10.1016/j.tics.2008.07.008 PMID:18805040

Smith, C. (1998). On authoritarianism in the dance classroom. Dance, power, and difference: Critical and feminist perspectives on dance education, 123-146. 
Smith, R. E., Smoll, F. L., \& Cumming, S. P. (2007). Effects of a motivational climate intervention for coaches on young athletes' sport performance anxiety. Journal of Sport \& Exercise Psychology, 29(1), 39-59. doi:10.1123/jsep.29.1.39 PMID:17556775

Stark, A., \& Newton, M. (2014). A dancer's wellbeing: The influence of the social psychological climate during adolescence. Psychology of Sport and Exercise, 15(4), 356-363. doi:10.1016/j.psychsport.2014.03.003

Steinberg, N., Siev-Ner, I., Peleg, S., Dar, G., Masharawi, Y., \& Hershkovitz, I. (2008). Growth and development of female dancers aged 8-16 years. American Journal of Human Biology, 20(3), 299-307. doi:10.1002/ajhb.20718 PMID:18203124

Stoeber, J. (2014). Perfectionism in sport and dance: A double-edged sword. International Journal of Sport Psychology, 45(4), 385-394.

Sugar, M. (1993). Female Adolescent Development (2nd ed.). New York: Brunner/Mazel.

Summers-Effler, E. (2004). Little girls in women's bodies: Social interaction and the strategising of early breast development. Sex Roles, 51(1-2), 29-44. doi:10.1023/B:SERS.0000032307.16204.ec

Tanchev, P. I., Dzherov, A. D., Parushev, A. D., Dikov, D. M., \& Todorov, M. B. (2000). Scoliosis in rhythmic gymnasts. Spine, 25(11), 1367-1372. doi:10.1097/00007632-200006010-00008 PMID:10828918

Treloar, S. A., \& Martin, N. G. (1990). Age at menarche as a fitness trait: Nonadditive genetic variance detected in a large twin sample. American Journal of Human Genetics, 47(1), 137. PMID:2349942

Ulijaszek, S. J., Johnston, F. E., \& Preece, M. A. (Eds.). (1998). The Cambridge encyclopaedia of human growth and development. Cambridge University Press.

Vani, M. F., Pila, E., \& Sabiston, C. M. (2017). Understanding sport dropout: Exploring the experiences of adolescent girls with body image concerns. Journal of Exercise, Movement, and Sport, 49(1), 138.

Wainwright, S. P., Williams, C., \& Turner, B. S. (2006). Varieties of habitus and the embodiment of ballet. Qualitative Research, 6(4), 535-558. doi:10.1177/1468794106068023

Walker, I. J., \& Nordin-Bates, S. M. (2010). Performance anxiety experiences of professional ballet dancers the importance of control. Journal of Dance Medicine \& Science: Official Publication of the International Association for Dance Medicine \& Science, 14(4), 133-145. PMID:21703084

Warren, M. (1989). Reproductive function in the ballet dancer. In K. M. Pirke, W. Wuttke \& U. Schweiger (Eds.), The MenstrualCycle and Its Disorders (pp. 161-170). Springer. doi:10.1007/978-3-642-74631-4_17

Warren, M. P., Gunn, J. B., Hamilton, L. H., Warren, L. F., \& Hamilton, W. G. (1986). Scoliosis and fractures in young ballet dancers. The New England Journal of Medicine, 314(21), 1348-1353. doi:10.1056/ NEJM198605223142104 PMID:3451741

Wiklund, M., Bengs, C., Malmgren-Olsson, E. B., \& Öhman, A. (2010). Young women facing multiple and intersecting stressors of modernity, gender orders and youth. Social Science \& Medicine, 71(9), 1567-1575. doi:10.1016/j.socscimed.2010.08.004 PMID:20846769 
Worthman, C. M., \& Trang, K. (2018). Dynamics of body time, social time and life history at adolescence. Nature, 554(7693), 451-457. doi:10.1038/nature25750 PMID:29469099

Wyatt, H. (2015). Physical development contributions to biomechanical injury risk in female gymnasts. Cardiff Metropolitan University.

\section{ADDITIONAL READING}

Barker-Ruchti, N., Kerr, R., Schubring, A., Cervin, G., \& Nunomura, M. (2017). "Gymnasts are like wine, they get better with age": Becoming and developing adult women's artistic gymnasts. Quest, 69(3), 348-365. doi:10.1080/00336297.2016.1230504

Cumming, S. P., Eisenmann, J. C., Smoll, F. L., Smith, R. E., \& Malina, R. M. (2005). Body size and perceptions of coaching behaviors by adolescent female athletes. Psychology of Sport and Exercise, 6(6), 693-705. doi:10.1016/j.psychsport.2005.01.002

Cumming, S. P., Standage, M., Loney, T., Gammon, C., Neville, H., Sherar, L. B., \& Malina, R. M. (2011). The mediating role of physical self-concept on relations between biological maturity status and physical activity in adolescent females. Journal of Adolescence, 34(3), 465-473. doi:10.1016/j.adolescence.2010.06.006 PMID:20655102

Hoyt, L. T., Niu, L., Pachucki, M. C., \& Chaku, N. (2020). Timing of puberty in boys and girls: Implications for population health. SSM - Population Health, 10, 100549. doi:10.1016/j.ssmph.2020.100549 PMID:32099893

McMahon, J., \& Barker-Ruchti, N. (2017). Assimilating to a boy's body shape for the sake of performance: Three female athletes' body experiences in a sporting culture. Sport Education and Society, 22(2), 157-174. doi:10.1080/13573322.2015.1013463

Mitchell, S. B. (2018). Psychological implications of puberty in dance. Accessed via https://www. onedanceuk.org/resource/growing-dancer-psychological-physiological-challenges-siobhan-mitchell/

Mitchell, S. B. (2018). The growing dancer: Physiological challenges. Accessed via https://www.onedanceuk.org/resource/growing-dancer-psychological-physiological-challenges-siobhan-mitchell/

Sherar, L. B., Cumming, S. P., Eisenmann, J. C., Baxter-Jones, A., \& Malina, R. M. (2010). Adolescent biological maturity and physical activity: Biology meets behavior. Pediatric Exercise Science, 22(3), 332-349. doi:10.1123/pes.22.3.332 PMID:20814031

Skoog, T., \& Stattin, H. (2014). Why and under what contextual conditions do early-maturing girls develop problem behaviors? Child Development Perspectives, 8(3), 158-162. doi:10.1111/cdep.12076

Yuan, A. S. V. (2012). Perceived breast development and adolescent girls' psychological wellbeing. Sex Roles, 66(11-12), 790-806. doi:10.100711199-012-0138-2 


\section{KEY TERMS AND DEFINITIONS}

Adolescence: The period of physical, cognitive and social maturation between childhood and adulthood. Encompasses development from age 10-11 years through to the early twenties.

Cognitive: Connected with thinking or conscious mental processes; relating to or involving the processes of thinking and reasoning - How an individual perceives and rationalises things.

Development: The study of change across the lifespan.

Maturation: The process of becoming mature. More specifically describes the development of three main biological systems: Sexual maturity, skeletal maturity, somatic maturity. Defined in terms of stage, tempo and timing (i.e., early, on-time, late)

Puberty: The process of physical changes resulting in adult proportions and functions.

Socio-Psychological: The psychological development of the individual in relation to his or her social environment. 